\title{
Clinically relevant magnetic resonance imaging (MRI) findings in elite swimmers' shoulders
}

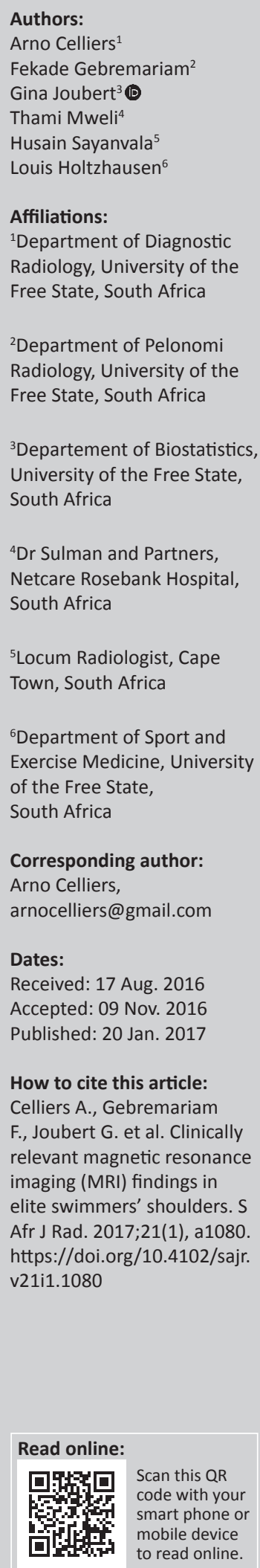

Background: Shoulder pain is the most common and well-documented site of musculoskeletal pain in elite swimmers. Structural abnormalities on magnetic resonance imaging (MRI) of elite swimmers' symptomatic shoulders are common. Little has been documented about the association between MRI findings in the asymptomatic shoulder versus the symptomatic shoulder.

Objective: To assess clinically relevant MRI findings in the shoulders of symptomatic and asymptomatic elite swimmers.

Method: Twenty (aged 16-23 years) elite swimmers completed questionnaires on their swimming training, pain and shoulder function. MRI of both shoulders $(n=40)$ were performed and all swimmers were given a standardised clinical shoulder examination.

Results: Both shoulders of 11 male and 9 female elite swimmers $(n=40)$ were examined. Eleven of the 40 shoulders were clinically symptomatic and 29 were asymptomatic. The most common clinical finding in both the symptomatic and asymptomatic shoulders was impingement during internal rotation, with impingement in $54.5 \%$ of the symptomatic shoulders and in $31.0 \%$ of the asymptomatic shoulders. The most common MRI findings in the symptomatic and asymptomatic shoulders were supraspinatus tendinosis (45.5\% vs. 20.7\%), subacromial subdeltoid fluid $(45.5 \%$ vs. $34.5 \%)$, increased signal in the AC Joint (45.5\% vs. $37.9 \%$ ) and AC joint arthrosis (36.4\% vs. $34.5 \%)$. Thirty-nine $(97.5 \%)$ of the shoulders showed abnormal MRI features.

Conclusion: MRI findings in the symptomatic and asymptomatic shoulders of young elite swimmers are similar and care should be taken when reporting shoulder MRIs in these athletes. Asymptomatic shoulders demonstrate manifold MRI abnormalities that may be radiologically significant but appear not to be clinically significant.

\section{Introduction}

Shoulder pain is the most common musculoskeletal complaint in competitive swimmers. ${ }^{1,2}$ Competitive swimmers registered with the USA Swimming national governing body average a distance of $60-80 \mathrm{~km}$ per week and 1.56 million overhead rotations per year. ${ }^{3}$ Both the stroke volume and multiple overhead rotations place tremendous stresses on the rotator cuff and glenohumeral joint, eventually resulting in the 'swimmer's shoulder' ${ }^{3,4}$ Arthroscopic findings suggest that the term swimmer's shoulder covers a variety of pathologies, including labral wearing and subacromial impingement. ${ }^{5}$ Connor et al. hypothesised that asymptomatic dominant shoulders of elite overhead athletes may have a higher incidence of magnetic resonance imaging (MRI) abnormalities than either their non-dominant shoulders or shoulders of asymptomatic volunteers. ${ }^{6}$

Elite swimmers demonstrate MRI changes of the shoulder similar to the imaging changes found on MRIs of persons with painful clinical syndromes. ${ }^{2}$ There seems to be limited information regarding MRI findings in overhead athletes who are asymptomatic. It has been suggested that these findings may be unique to overhead athletes, and care must be taken to correlate clinical history and physical examination with MRI findings in these patients with symptoms, as these changes might have been present premorbidly. ${ }^{2}$ Therefore, symptoms should not necessarily be attributed to the MRI changes. In a study by Sein et al., $84 \%$ of the swimmers studied had a positive impingement sign and $69 \%$ of those demonstrated supraspinatus tendinopathy on MRI. They concluded that supraspinatus tendinopathy was the major cause of shoulder pain in elite swimmers and this was induced by intensive swimming training. ${ }^{4}$ However, it was not possible to draw conclusions in the asymptomatic shoulders, as this portion of the studied group only constituted $9 \%$ of the total sample.

Copyright: (c) 2017. The Author(s). Licensee: AOSIS. This work is licensed under the Creative Commons Attribution License 
Fredericson et al. evaluated asymptomatic overhead athletes and all of the athletes studied had MRI findings that could be considered abnormal, despite the fact that they were clinically asymptomatic. ${ }^{2}$ They concluded that MRI changes could be interpreted as evidence of a previous subclinical injury unknown to the athlete and that perhaps accumulation of multiple micro traumas has an additive effect in the absence of a significant single injury. ${ }^{2}$ Our study serves to underline the important message that findings on imaging always need to be correlated with symptoms, as radiological abnormalities often do not correspond with clinical problems.

Screening or pre-injury MRI is rarely available for comparison when an athlete develops clinical symptoms. An improved understanding of the baseline signal changes in a pre-injury setting may lead to targeted injury-prevention training strategies. ${ }^{7}$ Miniaci et al. advocated baseline shoulder MRI for all baseball pitchers owing to the difficulty in interpreting signal changes in these athletes. ${ }^{7}$ Improved understanding of subclinical or clinically irrelevant findings in the overhead athlete may decrease the number of unnecessary medical or surgical interventions.

According to the researcher's literature search, this study is one of the largest studies investigating both shoulders in the symptomatic as well as the asymptomatic elite swimming population (Table 1).

\section{Research method and design}

For this cross-sectional study, the study population was voluntarily selected from the University of the Free State's swimming team located in Bloemfontein, South Africa. The clinical examinations were conducted at the Department of Sport and Exercise Medicine at the University of the Free State and the imaging (MRI) was done at the Pelonomi Regional Hospital in Bloemfontein.

The sample included 20 volunteers, both male and female elite swimmers between the ages of 16 and 25 years, with symptomatic and asymptomatic shoulders. An elite swimmer was defined as a swimmer with the ability to perform a $100 \mathrm{~m}$ freestyle race at or faster than $75 \%$ of a national record swimming time. ${ }^{8}$ Exclusion criteria were: previous shoulder surgery, previous fracture of the shoulder, inability or unwillingness to participate in the MRI and clinical shoulder examination. None of the swimmers were excluded from the study.
A standardised, self-administered swimming training questionnaire was completed by each study participant on the day of the MRI study. Descriptive characteristics were obtained for each swimmer using a standardised Shoulder Service Questionnaire. ${ }^{4}$ Items pertaining to the subject included age, gender, birth date, occupation, arm dominance (right, left or ambidextrous) and an overview of general health. Clinical parameters of the shoulder condition included the affected shoulder (right, left or both), date of injury onset, mechanism of onset (whether traumatic or insidious).

Frequency and severity of the shoulder pain, stiffness of the shoulder, difficulty in reaching behind the back, difficulty with activities above the head, overall shoulder status, current level of activity and highest level of sport at the time of examination were obtained by the sports physician in the clinical shoulder questionnaire.

All of the 20 participants ( $n=40$ shoulders) were examined clinically by a sports physician (M Phil (Sports Medicine), University of Cape Town), with a special interest in the swimmer's shoulder. The clinical examination included examination of shoulders (symptomatic and asymptomatic), rotator cuff tests, impingement tests, instability tests as well as evaluation for local tenderness (sternoclavicular joint, acromioclavicular joint, biceps, subacromial). The findings of these tests were recorded on a standardised clinical form.

\section{Materials}

The MRIs were performed, using a MAGNETOM Aera 1.5-tesla MRI scanner (Siemens), utilising high-resolution technique with a dedicated shoulder coil in a standardised protocol with fixed sequence parameters (time of repetition, time of echo, slice thickness, matrix, field of view).

\section{Procedure}

MRI without arthrography was performed on both shoulders of all 20 participants $(n=40)$ with the arm placed in adduction and neutral rotation. The following sequences were performed: (1) Coronal plane: T2-weighted FSE and a PD FSE sequence with fat saturation; (2) Sagittal oblique plane: T2-weighted FSE and a PD FSE sequence with fat saturation; (3) Axial plane: T2* GRE and a PD FSE sequence with fat saturation.

The images were reviewed by three consultant radiologists, one with a musculoskeletal radiology subspecialty and two

TABLE 1: Comparison with similar studies.

\begin{tabular}{|c|c|c|c|c|c|c|c|}
\hline Date & Source of study & MRI sample size & Sample population & Mean age (years) & Asymptomatic (\%) & Symptomatic (\%) & Unilateral or Bilateral MRI \\
\hline 2016 & This study & 40 & Elite swimmers & 18.9 & 72.5 & 27.5 & Bilateral \\
\hline 2014 & Klein et al. (10) & 56 & Water polo & 24 & 100.0 & 0.0 & Bilateral \\
\hline 2010 & Sein et al. (5) & 52 & Elite swimmers & 16 & 9.0 & 91.0 & Unilateral \\
\hline 2009 & Fredericson et al. (2) & 33 & $\begin{array}{l}\text { Swimmers }(\mathrm{n} 6) \text {, volley ball } \\
\text { players and gymnasts }\end{array}$ & 19.6 & 100.0 & 0.0 & Unilateral \\
\hline 2008 & Reuter et al. (11) & 23 & Triathletes & 37 & 30.4 & 69.6 & Unilateral \\
\hline 2003 & Connor et al. (7) & 40 & $\begin{array}{l}\text { Tennis players and baseball } \\
\text { pitchers }\end{array}$ & 26.4 & 100.0 & 0.0 & Bilateral \\
\hline 2002 & Miniaci et al. (9) & 28 & Baseball pitchers & 20.1 & 100.0 & 0.0 & Bilateral \\
\hline
\end{tabular}

MRI, magnetic resonance imaging. 
with experience in musculoskeletal radiology. The radiologists were blinded to grouping, dexterity, as well as clinical history and examination. Their findings were documented on a standardised self-administered assessment form that was compiled from Stoller's textbook of MRI in orthopaedics and sports medicine. ${ }^{9}$ The checklist consisted of 135 variables that had to be evaluated in the three different planes. There was some overlap in the different planes between the anatomical structures that had to be evaluated, and the findings of the same anatomical structures were collated in the statistical analysis of the data. The following anatomical structures had to be evaluated and commented on: Coronal plane (1) rotator cuff, (2) AC joint, (3) biceps tendon (longhead), (4) labrum, (5) IGHL, (6) glenohumeral joint cartilage, (7) osseous structures (humeral head, glenoid, scapula, suprascapular notch), (8) deltoid, (9) subacromial subdeltoid bursa; Axial plane: (1) AC joint, (2) subscapularis and biceps tendon, (3) labrum, (4) joint capsule structures, (5) glenohumeral joint cartilage, (6) osseous structures (Hill-Sachs deformities, posterolateral humeral head, glenoid, coracoid process, spinoglenoid notch), (7) supraspinatus and infraspinatus tendons, (8) pectoralis major muscle and deltoid; Sagittal oblique plane: (1) rotator cuff, (2) acromion, (3) AC joint, (4) rotator interval, biceps tendon, coracohumeral ligament and SGHL, (5) glenoid fossa, (6) MGHL,IGHL, (7) subacromial subdeltoid bursa. ${ }^{9}$

For each item, a yes or no had to be indicated by the radiologists. For analysis, consensus between the radiologists was used (i.e. the answer given by two or more of the radiologists).

\section{Analyses}

Results were summarised by frequencies and percentages (categorical variables) and means, standard deviations or percentiles (numerical variables). Statistical comparison of subgroups was done using Fisher's exact test owing to sparse cells. $P$-values of less than 0.05 were considered statistically significant.

\section{Ethical considerations}

The study was conducted in accordance with the principles of the Declaration of Helsinki and Good Clinical practice guidelines. ${ }^{10}$ All participants signed an informed consent form and consent was obtained from legal guardians for participants younger than 18 years. The Ethics Committee of the Faculty of Health Sciences, University of the Free State approved the study, with ethical clearence number: ECUFS NR 117/2014.

\section{Results}

The demographics, symptoms and clinical examination were compared with the methods and questions as performed in a previous study evaluating elite swimmers. ${ }^{4}$

\section{Demographics of the swimmers}

Eleven (55\%) of the elite swimmers were male and 9 (45\%) were female. The mean age was 18.9 years and all swimmers had been coached for at least 5 years, with a median time of 11.5 years. Nineteen swimmers (95\%) represented their country at an international level and one swimmer (5\%) was competing at the national level. Median training time spent on a specific stroke was $60 \%$ freestyle, $14 \%$ butterfly stroke, $12.5 \%$ backstroke and $20 \%$ breaststroke. The median time the swimmers practised in the water was $13.5 \mathrm{~h} /$ week (range: $3 \mathrm{~h} /$ week - $18 \mathrm{~h} /$ week) and the median distance swum was $40 \mathrm{~km} /$ week (range: $9 \mathrm{~h} /$ week - $60 \mathrm{~km} /$ week).

\section{Clinical symptoms}

Of the 40 shoulders studied, 11 (27.5\%) were symptomatic. Two swimmers ( $n=4$ shoulders) reported bilateral shoulder pain and 7 ( $n=7$ shoulders) reported unilateral shoulder pain. All 11 symptomatic shoulders were painful during activity (27.3\% always, $18.2 \%$ daily, $27.3 \%$ weekly and $27.3 \%$ monthly). In 2 of the 11 symptomatic shoulders (18.8\%), severe pain with activities above their head was stated. In $6(54.6 \%)$ of the 11 symptomatic shoulders, the complaint was that of a stiff shoulder (severe: $36.4 \%$, moderate: $9.1 \%$, mild: $9.1 \%$ ). The participants with symptomatic shoulders ( $n=11$ shoulders) were asked to grade their shoulder pain into none, mild, moderate, severe or very severe. In five shoulders (45.5\%), the self-assessed shoulder pain was found to be mild. All symptomatic shoulders $(n=11)$ received physiotherapy, $9(81.8 \%)$ previously received corticosteroid injections and $8(72.7 \%)$ previously received acupuncture.

\section{Clinical examination of the shoulder}

Of the 40 shoulders examined, the most common positive findings were: a positive impingement sign in internal rotation elicited with the Hawkins-Kennedy test $(n=15$; $37.5 \%)$, positive $\mathrm{O}^{\prime}$ Brien's sign $(n=12 ; 30.0 \%)$, biceps tenderness $(n=11 ; 27.5 \%)$, as well as AC joint tenderness ( $n=9$; $22.5 \%)$. Sternoclavicular tenderness $(18.2 \%$ vs. $17.2 \%)$ was slightly more common in the symptomatic group.

None of the shoulders demonstrated muscle wasting of the rotator cuff or supporting muscles. Of the 11 symptomatic shoulders, one $(9.1 \%)$ had reduced power of four out of five for rotator cuff strength testing, as well as mild anterior instability. None of the asymptomatic shoulders demonstrated loss of power or instability. Details regarding the clinical examination findings in swimmers are shown in Table 2.

TABLE 2: Clinical examination findings.

\begin{tabular}{|c|c|c|c|c|}
\hline \multirow[b]{3}{*}{ Clinical findings } & \multicolumn{4}{|c|}{ Positive } \\
\hline & \multicolumn{2}{|c|}{ Symptomatic shoulder $\dagger$} & \multicolumn{2}{|c|}{ Asymptomatic shoulder: } \\
\hline & $n$ & $\%$ & $n$ & $\%$ \\
\hline Drop arm & 0 & 0 & 1 & 3.4 \\
\hline Impingement (ER) & 2 & 18.2 & 3 & 10.3 \\
\hline Impingement (IR) & 6 & 54.5 & 9 & 31.0 \\
\hline Apprehension & 2 & 18.2 & 4 & 13.8 \\
\hline O' Brien's sign & 6 & 54.5 & 6 & 20.7 \\
\hline Paxinos & 0 & 0 & 1 & 3.4 \\
\hline SC joint tenderness & 2 & 18.2 & 5 & 17.2 \\
\hline AC joint tenderness & 6 & 54.5 & 3 & 10.3 \\
\hline Biceps tenderness & 5 & 45.5 & 6 & 20.7 \\
\hline Subacromial tenderness & 3 & 27.3 & 3 & 10.3 \\
\hline
\end{tabular}

$\dagger, n=11 ; \ddagger, n=29$. 


\section{Magnetic resonance imaging findings}

Of the 29 asymptomatic shoulders studied, 25 (86.2\%) had MRI changes which could be viewed as abnormal. Ten (91\%) of the 11 symptomatic shoulders had abnormal MRI findings. The most common MRI findings (Figures 1-3) in the symptomatic shoulders were supraspinatus partial tear $(45.5 \%$ in symptomatic shoulders vs. $20.7 \%$ in asymptomatic shoulders), subacromial subdeltoid fluid (45.5\% vs. $34.5 \%)$, increased signal in the AC Joint (45.5\% vs. 37.9\%), supraspinatus tendinosis (36.4\% vs. $10.3 \%$ ) and AC joint arthrosis (36.4\% vs. $34.5 \%)$. Supraspinatus tendinosis (36.4\% vs. $10.3 \%$ ) and partial tear of the supraspinatus ( $45.5 \%$ vs. $20.7 \%$ ) were much more common in the symptomatic shoulders compared with the asymptomatic shoulders. Biceps tendinosis was common in both symptomatic and asymptomatic shoulders (18.2\% vs. $6.9 \%)$. Degeneration in the proximal bicipital groove $(18.2 \% \mathrm{vs}$. $0 \%$ ) was more common in the symptomatic population. Thickening of the inferior glenohumeral ligament was more common in the asymptomatic shoulders ( $13.8 \%$ vs. $0.0 \%)$. Increased signal of the labrum and labral tears were comparable in the two groups $(10.0 \%$ vs. $10.3 \%)$.

Twenty (50\%) of the shoulders in this study $(n=40)$ had a positive impingement sign with the Hawkins-Kennedy impingement test. Of these, $8(72.7 \%)$ were clinically symptomatic shoulders $(n=11)$ and $12(41.4 \%)$ were clinically asymptomatic shoulders $(n=29)$. In both groups, $25 \%$ of the shoulders had positive clinical signs of impingement that were correlated with MRI features of supraspinatus tendinosis (2 of 8 asymptomatic, 3 of 12 symptomatic).
In the clinically symptomatic group $(n=11)$, three $(27.3 \%)$ shoulders tested negative for impingement but still demonstrated MRI features consistent with supraspinatus tendinosis. In the clinically asymptomatic group $(n=29), 17$ (58.6\%) tested negative for impingement clinically, but only four of these $(23.5 \%)$ demonstrated MRI features consistent with supraspinatus tendinopathy.

\section{Discussion}

Shoulder pain in overhead athletes is a common problem owing to tremendous repetitive stresses. ${ }^{6}$

We examined elite swimmers clinically and with bilateral shoulder MRI. Most of these young elite swimmers aspire to compete at the senior international level. They spend on average 13.5 hours a week in the pool and swim an average of $40 \mathrm{~km} /$ week. The emphasis of the study was on differentiating clinically insignificant MRI findings in elite athletes from those that are clinically and radiologically significant (Figure 4). It is paramount to provide a baseline of what might be considered clinically irrelevant signal changes and findings in this population, in order to reduce further morbidity by limiting over-diagnosis and preventing unnecessary surgical interventions. Miniaci et al. advocated baseline shoulder MRI for all baseball pitchers owing to the difficulty in interpreting signal changes in these athletes. ${ }^{7}$

We aimed to evaluate the overlap of MRI findings in the symptomatic and asymptomatic shoulders in order to

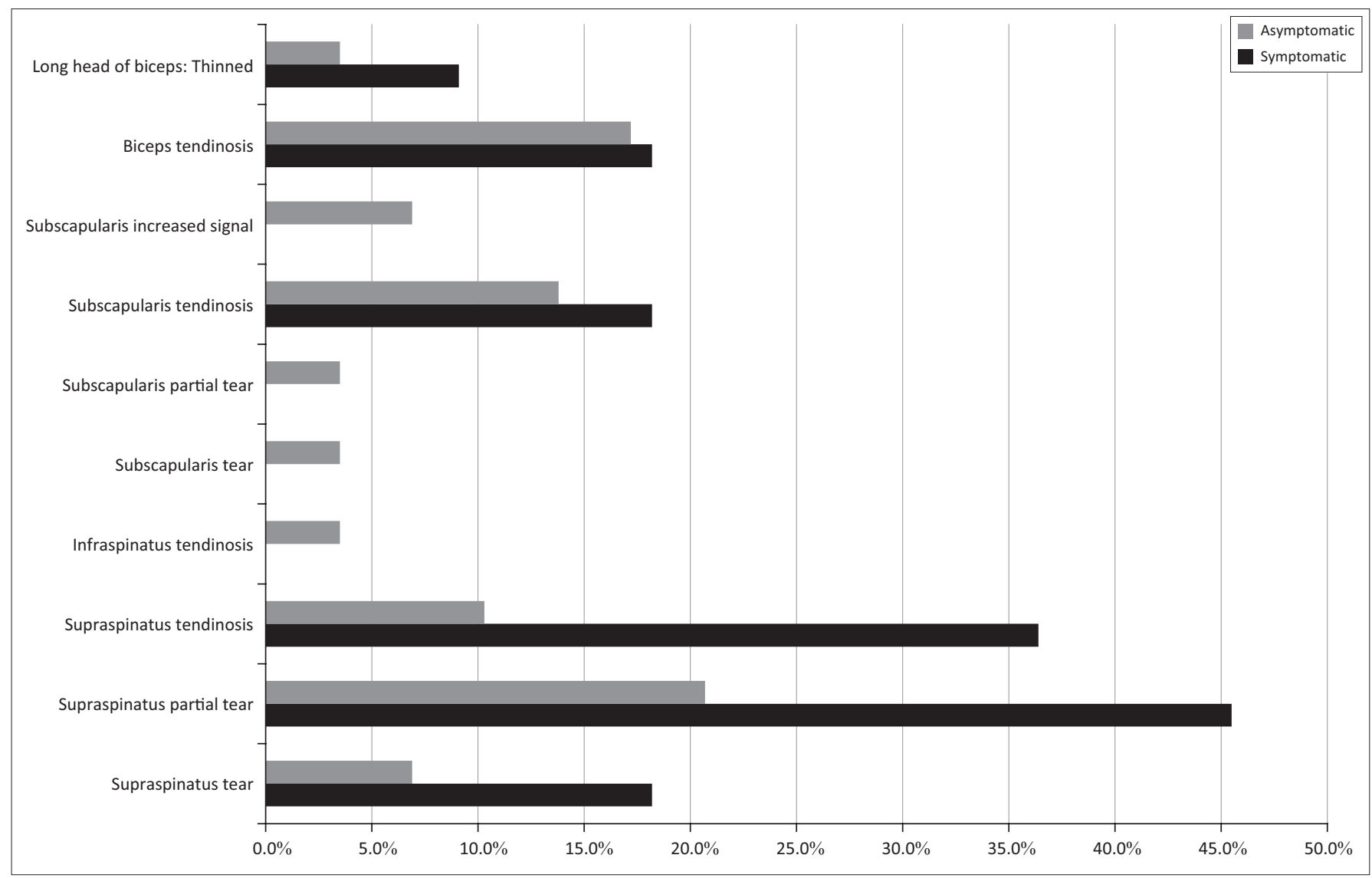

FIGURE 1: Magnetic resonance imaging findings in the rotator cuff. 


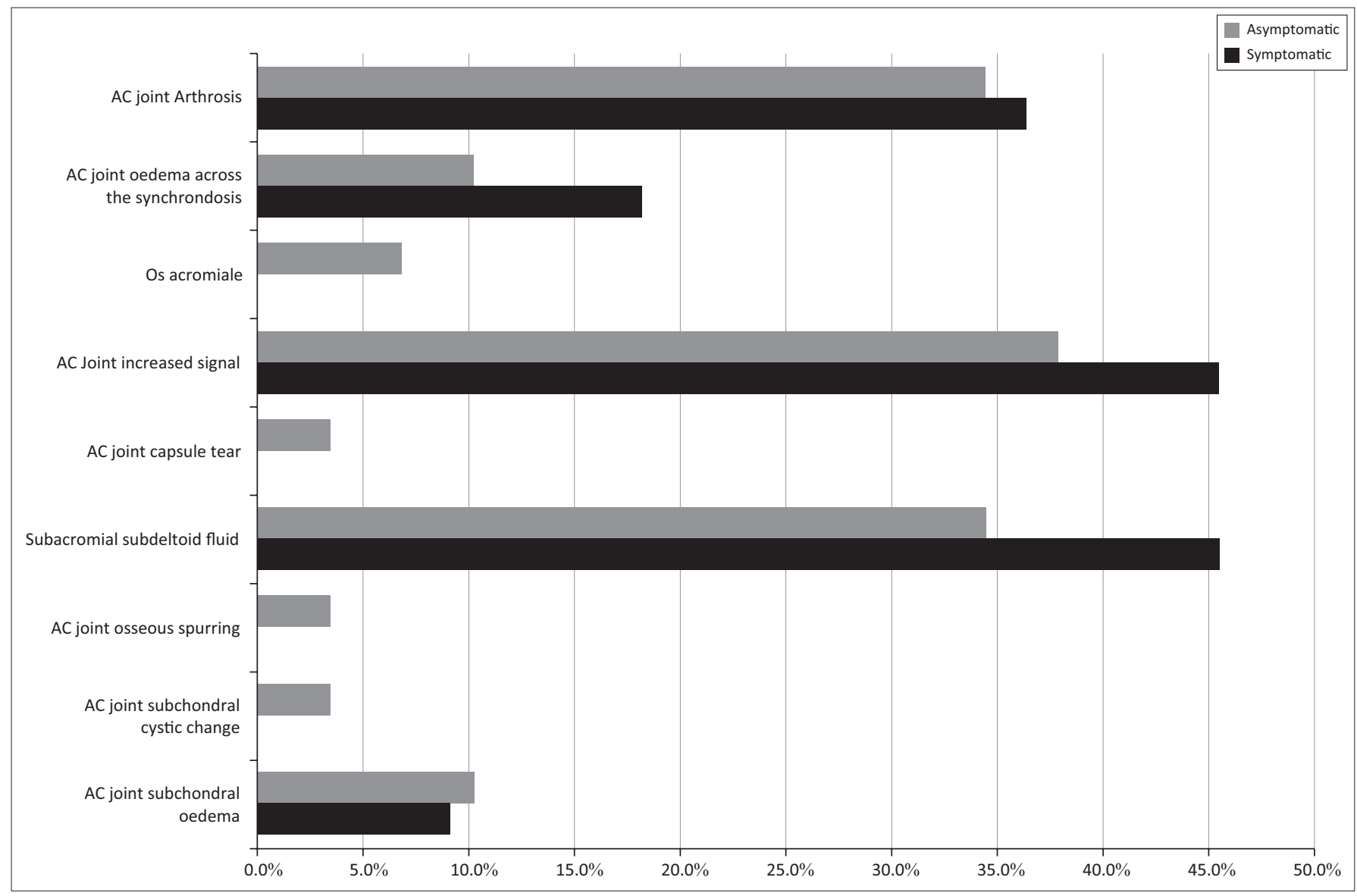

FIGURE 2: Magnetic resonance imaging findings in and around the AC Joint.

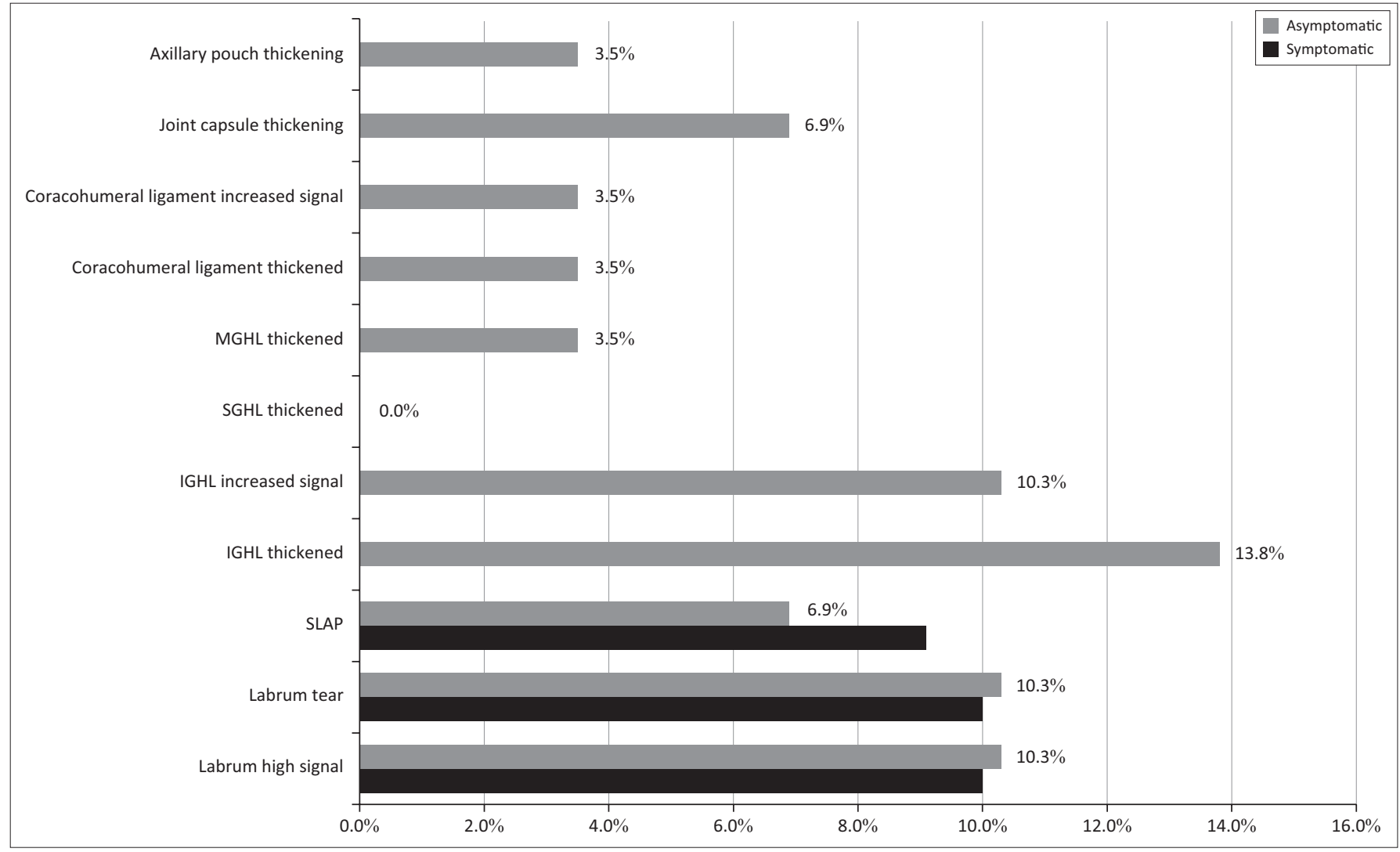

FIGURE 3: Magnetic resonance imaging findings in the joint capsule. 
elucidate the fact that asymptomatic shoulders may demonstrate manifold MRI abnormalities that may be radiologically significant but appear not to be clinically significant.

Of the asymptomatic shoulders studied, $86.2 \%$ had MRI changes which could be viewed as abnormal. This corresponds to previous studies that showed that MRI abnormalities in asymptomatic overhead athletes are common. ${ }^{2,411}$ The most common findings in both the symptomatic and asymptomatic shoulders were subacromial subdeltoid fluid (Figure 5), increased signal at the AC joint (Figure 6), AC joint arthrosis, supraspinatus tendinosis (Figure 7) and biceps tendinosis (Figure 8). There were 11 symptomatic shoulders in our sample group with the most common clinical findings being impingement, AC joint tenderness, positive $\mathrm{O}^{\prime}$ Brien's sign and biceps tenderness. The asymptomatic group also demonstrated positive clinical findings, with subacromial impingement, biceps tenderness

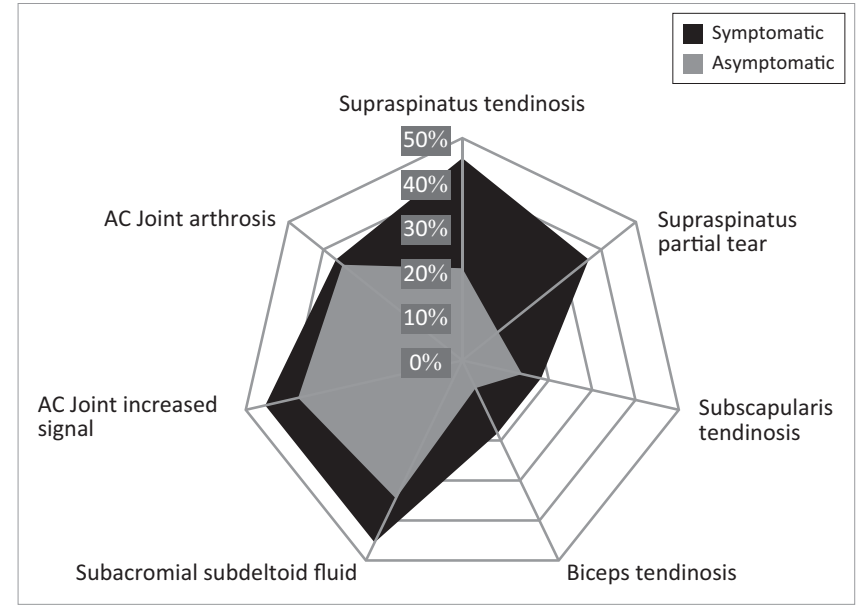

FIGURE 4: Summary of the most common magnetic resonance imaging findings.

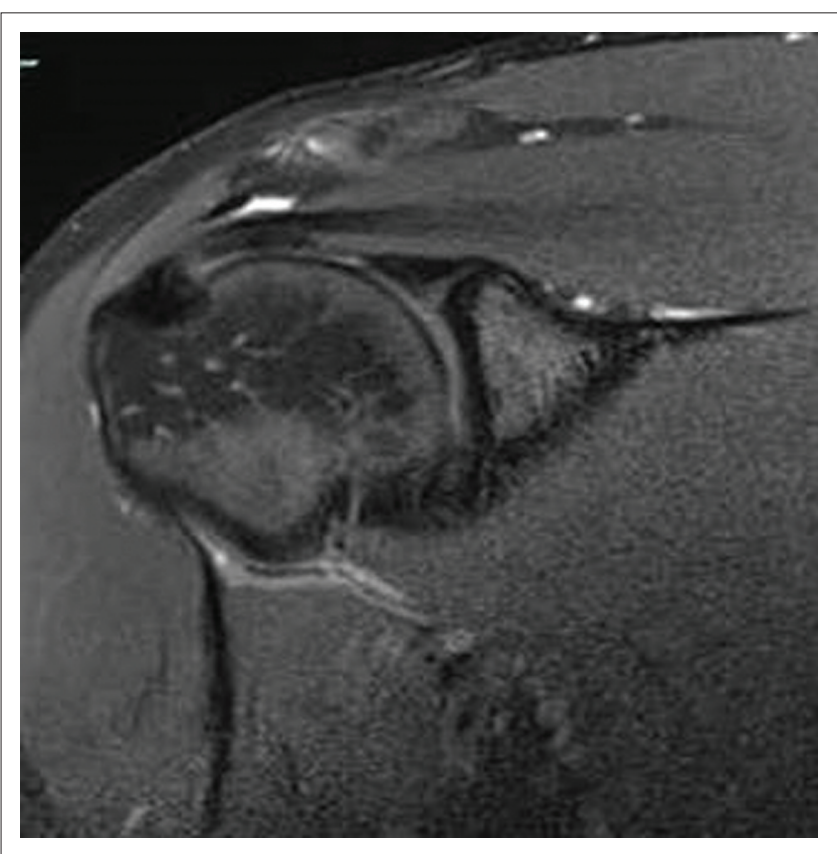

FIGURE 5: Subacromial subdeltoid fluid in the asymptomatic dominant shoulder of a 16 -year-old swimmer. and sternoclavicular tenderness being the most common findings. AC Joint tenderness was less common in the asymptomatic group. The studied group consisted of a slightly smaller symptomatic group than Sein et al., but our athletes more frequently demonstrated local AC joint tenderness and positive O'Brien's sign. Increased signal at the $\mathrm{AC}$ joint is a common finding in both the symptomatic and asymptomatic shoulders of overhead athletes and does not correlate with patient symptomatology as also noted in agreement with Reuter et al. ${ }^{12}$ In this study, type 1 acromion

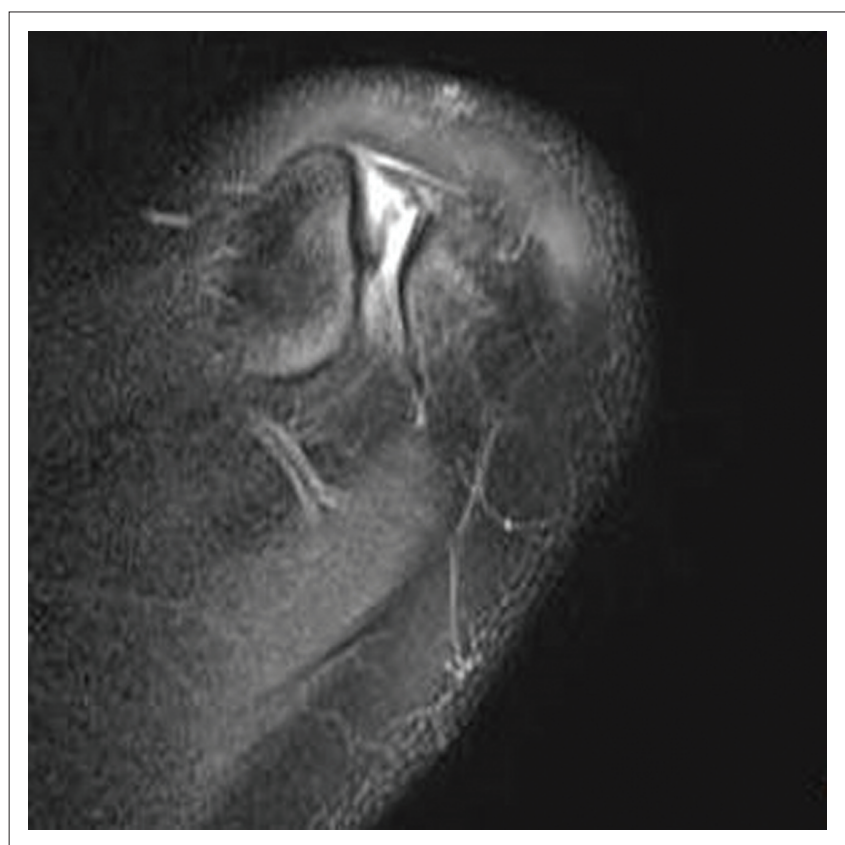

FIGURE 6: Increased signal in the AC joint in the asymptomatic non-dominant shoulder of a 17-year-old swimmer.

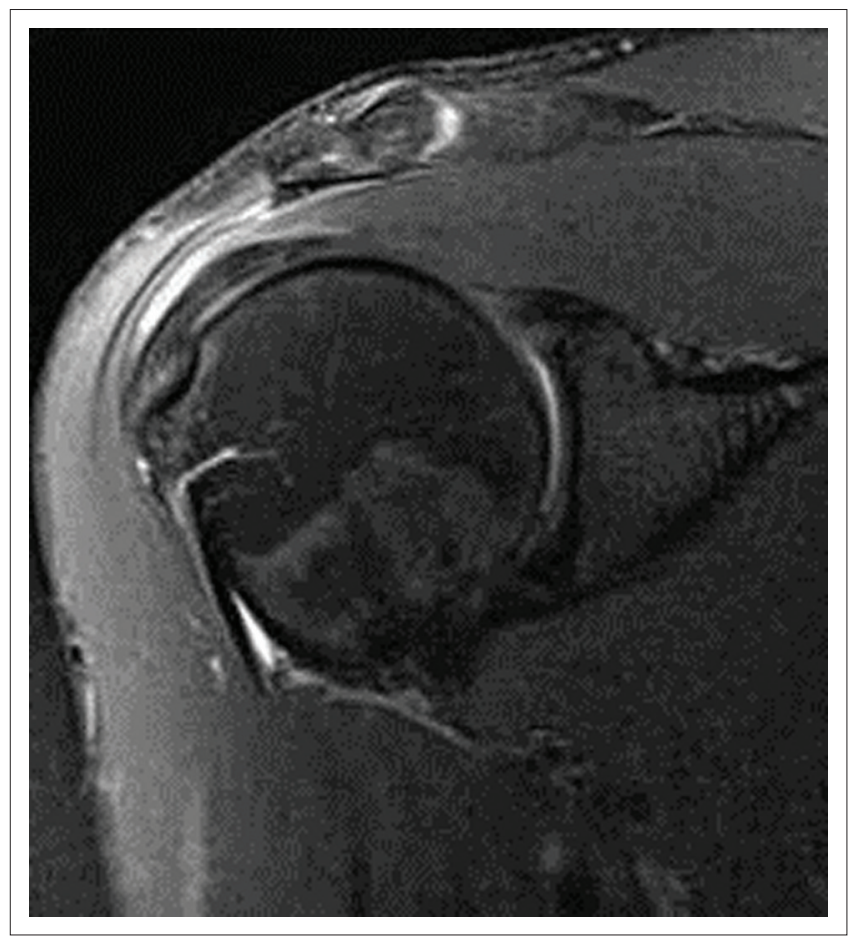

FIGURE 7: Supraspinatus tendinosis in the asymptomatic non-dominant shoulder of a 19-year-old swimmer. 


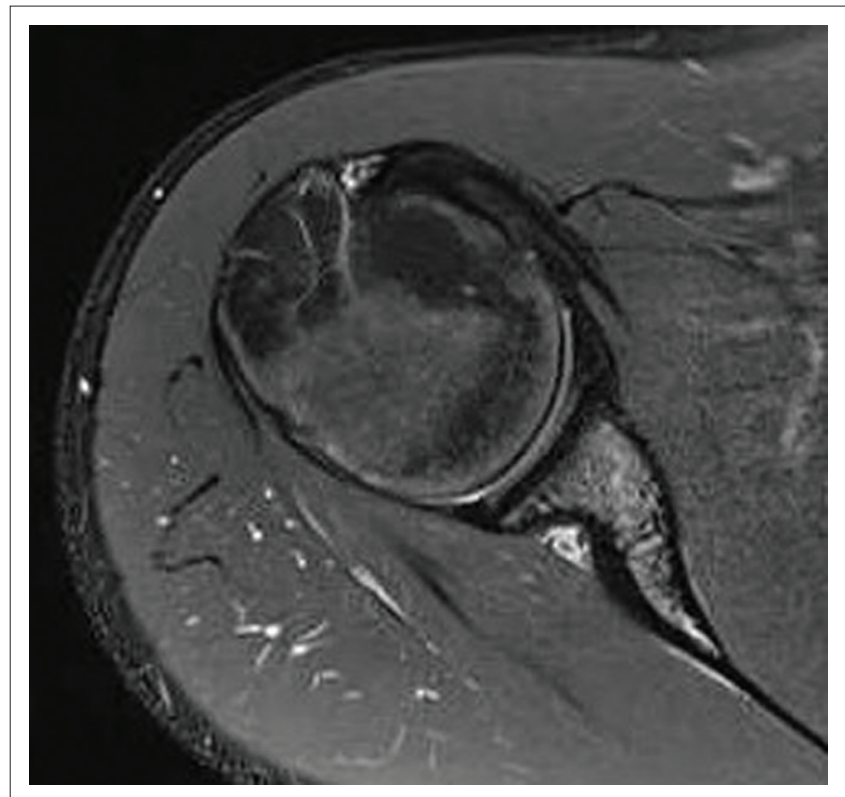

FIGURE 8: Fluid in the bicipital groove in the asymptomatic dominant shoulder of a 17 -year-old swimmer.

shape was most common followed by type 2 and type 3 . There was no direct correlation between shoulder pain and acromion shape.

Supraspinatus tendinopathy was a common finding in both the symptomatic and asymptomatic shoulders, with it being nearly twice as common in the symptomatic shoulders. Previous studies also found supraspinatus tendinopathy to be a dominant finding. ${ }^{4}$ Biceps tendinosis was approximately twice as common in the symptomatic shoulder. Connor et al. showed that subacromial subdeltoid fluid was a common finding in the asymptomatic shoulder. ${ }^{6}$ This study demonstrated similar results with subacromial and/or subdeltoid fluid found in $34.5 \%$ of the asymptomatic shoulders. In a study where Reuter et al. evaluated shoulder MRI changes in 23 triathletes, $57 \%$ of the asymptomatic group and $31 \%$ of the symptomatic group demonstrated AC joint arthrosis. ${ }^{12}$ The current study demonstrated nearly equal percentages of AC joint arthrosis (36.4\% symptomatic vs. $34.5 \%$ asymptomatic).

Most of our findings correlate with previous studies done, demonstrating multiple abnormal MRI signal changes in the asymptomatic shoulder. ${ }^{4,11}$

\section{Limitations of the study}

The majority of the studied population was from the same training facility with the same coaching staff. Therefore, the analysis may suffer from sampling errors related to local factors and/or the fact that clinical symptoms were based on subjective self-reporting. The study sample consists of volunteers; therefore, there is a lack of randomisation. Another limitation is that previous studies ${ }^{7}$ referred to the possibility that owing to the young age of the athletes, some of the observed MRI findings might become symptomatic later in their professional career. Further studies with long-term follow-up are required. MRI was done without arthrography as complications could not be justified. Therefore, there is no arthroscopic correlation with MRI findings. Another possible limitation is that the study did not make use of T1W MR sequences. Sequences used were derived from Stoller textbook of muskulosceletal MRI, which did not include T1W sequences. Although it is not essential for this study, T1W sequences may be utilised in future studies.

\section{Conclusion}

The importance of this study lies in the fact that supraspinatus abnormalities on MRI are common in asymptomatic shoulders. Asymptomatic shoulders demonstrate manifold MRI abnormalities that may be radiologically significant but appear not to be clinically significant. The researcher suggests a pre-season baseline MRI for all elite swimmers with followup imaging if supraspinatus pathology was identified on the baseline MRI. An improved understanding of the baseline signal changes in a pre-injury setting may lead to targeted injury-prevention training strategies. Improved understanding of subclinical or clinically irrelevant findings in this population may lead to avoidance of unnecessary medical or surgical intervention.

\section{Acknowledgements}

The authors would like to thank Melvin, Sam and Joshua for performing the imaging.

\section{Competing interests}

The authors declare that they have no financial or personal relationships that may have inappropriately influenced them in writing this article.

\section{Authors' contributions}

F.G. was the project leader and A.C. was the lead author. G.J. made conceptual contributions and did the biostatistical analysis. L.H. performed the clinical examinations and also made conceptual contributions. H.S. and T.M. identified the necessity for this research analysis and assisted in the finalisation of this article.

\section{References}

1. Bak K. The practical management of swimmer's painful shoulder: Etiology, diagnosis, and treatment. Clin J Sport Med. 2010;20(5):386-390. http://doi. org/10.1097/JSM.0b013e3181f205fa

2. Fredericson M, Ho C, Waite B, Jennings F, Peterson J, Williams C, et al. Magnetic resonance imaging abnormalities in the shoulder and wrist joints of asymptomatic elite athletes. PM R. 2009;1(2):107-116. Available from: http://www. sciencedirect.com/science/article/pii/S193414820800018X

3. Heinlein SA, Cosgarea AJ. Biomechanical considerations in the competitive swimmer's shoulder. Sports Health. $2010 ; 2(6): 519-525$. Available from: http:// www.pubmedcentral.nih.gov/articlerender.fcgi?artid $=3438875 \&$ tool $=$ pmcentrez \&rendertype=abstract

4. Sein ML, Walton J, Linklater J, et al. Shoulder pain in elite swimmers: Primarily due to swim-volume-induced supraspinatus tendinopathy. Br J Sports Med. 2010;44(2):105-113. http://dx.doi.org/10.1136/bjsm.2008.047282

5. Brushøj C, Bak K, Johannsen HV, Faun $\varnothing$ P. Swimmers' painful shoulder arthroscopic findings and return rate to sports. Scand J Med Sci Sports. 2007;17(4):373-377. Available from: http://www.ncbi.nlm.nih.gov/pubmed/16805785 
6. Connor PM, Banks DM, Tyson AB, Coumas JS, D'Alessandro DF. Magnetic resonance imaging of the asymptomatic shoulder of overhead athletes: A 5-year follow-up study. Am J Sports Med. 2003;31(5):724-727.

7. Miniaci A, Mascia AT, Salonen DC, Becker EJ. Magnetic resonance imaging of the shoulder in asymptomatic professional baseball pitchers. Am J Sports Med. 2002;30(1):66-73.

8. Aujouannet YA, Bonifazi M, Hintzy F, Vuillerme N, Rouard AH. Effects of a highintensity swim test on kinematic parameters in high-level athletes. Appl Physio
Nutr Metab. 2006;31(1975):150-158. http://dx.doi.org/10.1139/h05-012

9. Stoller DW. Magnetic resonance imaging in orthopaedics and sports medicine. Philadelphia, PA: Lippincott Williams \& Wilkins; 2007.
10. World Medical Association. Declaration of Helsinki - Ethical principles for medical research involving human subjects [homepage on the Internet]. 2013 [cited 2015 Dec 7]. Available from: http://www.wma.net/en/30publications /10policies/b3/

11. Klein M, Tarantino I, Warschkow R, et al. Specific shoulder pathoanatomy in semiprofessional water polo players: A magnetic resonance imaging study. Orthop J Sport Med. 2014;2:1-6. http://dx.doi.org/10.1177/2325967114531213

12. Reuter RM, Hiller WD, Ainge GR, et al. Ironman triathletes: MRI assessment of the shoulder. Skeletal Radiol. 2008;37(8):737-741. http://dx.doi.org/10.1007/ s00256-008-0516-6 